\title{
Kétamine pour le traitement de la douleur aiguë
}

\author{
William K. Silverstein MD, David N. Juurlink MD PhD, Jonathan S. Zipursky MD
}

Citation : CMAJ 2021 November 1;193:E1663. doi : 10.1503/cmaj.210878-f

Voir la version anglaise de l'article ici : www.cmaj.ca/lookup/doi/10.1503/cmaj.210878

\section{Les effets analgésiques et anesthésiques de la kétamine sont dose-dépendants}

La kétamine est un dérivé de la phencyclidine (PCP) qui agit principalement en inhibant les récepteurs $N$-méthyl-D-aspartate ${ }^{1}$. En doses faibles $(0,1-0,3 \mathrm{mg} / \mathrm{kg})$, la kétamine produit surtout une analgésie. L'anesthésie dissociative et les effets psychomimétiques viennent avec des doses plus élevées $(\geq 1,0 \mathrm{mg} / \mathrm{kg})$.

2

La kétamine est un analgésique efficace lorsqu'elle est

administrée à des doses sous-anesthésiques

La kétamine à faible dose est un analgésique efficace dans plusieurs contextes ${ }^{2}$. La kétamine intraveineuse à des doses sous-anesthésiques réduit les scores de douleur postopératoire et prolonge l'intervalle avant une première demande d'analgésie de la part du patient ${ }^{3}$. Pour la douleur aiguë, la kétamine à faible dose exerce des effets analgésiques comparables à ceux de la morphine avec un besoin similaire d'analgésie de secours ${ }^{4}$. La kétamine peut être administrée par voie parentérale en perfusions intermittentes (pendant 10-15 min.) ou continues. Les médecins devraient d'abord prescrire une dose faible $(0,1 \mathrm{mg} / \mathrm{kg}$ toutes les $4 \mathrm{~h}$ pour une administration intermittente; $0,1 \mathrm{mg} / \mathrm{kg} / \mathrm{h}$ pour une perfusion continue) et augmenter au besoin jusqu'à concurrence de $0,35 \mathrm{mg} / \mathrm{kg}$ (intermittente) ou $0,25 \mathrm{mg} / \mathrm{kg} / \mathrm{h}$ (continue) $)^{2}$.

\section{3}

\section{La kétamine à faible dose est sécuritaire}

Les effets indésirables associés à la kétamine à dose plus élevée (nausées, vomissements, rêves d'apparence réelle, hallucinations, dissociation) ne surviennent pas plus souvent qu'avec le placebo lorsqu'elle est utilisée aux doses pour traiter la douleur ${ }^{2,3}$. Les études qui ont comparé la kétamine à faible dose à la morphine pour le traitement de la douleur aiguë ne font état d'aucune différence quant à l'incidence des effets indésirables ${ }^{4}$.

\section{Les médecins devraient vérifier les contre-indications avant d'amorcer un traitement}

La kétamine n'est pas recommandée chez les patients atteints de psychose, de maladie cardiovasculaire grave ou d'insuffisance hépatique ${ }^{2}$. Étant donné le manque de données sur l'innocuité durant la grossesse, il est suggéré aux médecins de consulter des experts s'ils envisagent de l'utiliser dans un tel contexte.

\section{L'utilisation de la kétamine pour le traitement de la douleur aiguë peut produire des effets d'épargne d'opioïdes}

Ajoutée aux opioïdes pour la prise en charge de la douleur aiguë, la kétamine permet de réduire les scores de douleur et la dose totale d'opioïdes sans augmenter la sédation ou les autres effets indésirables ${ }^{2}$. La dépression respiratoire associée aux opioïdes est également évitée. Les bienfaits peuvent perdurer au-delà du séjour hospitalier, car on a signalé une réduction de la consommation d'opioïdes après le congé chez les patients traités par kétamine intraveineuse en période périopératoire ${ }^{5}$.

\section{Références}

1. Hirota K, Lambert DG. Ketamine: New uses for an old drug? Br J Anaesth 2011;107:123-6.

2. Schwenk ES, Viscusi ER, Buvanendran A, et al. Consensus guidelines on the use of intravenous ketamine infusions for acute pain management from the American Society of Regional Anesthesia and Pain Medicine, the American Academy of Pain Medicine, and the American Society of Anesthesiologists. Reg Anesth Pain Med 2018;43:456-66.

3. Brinck E, Tiippana E, Heesen M, et al. Perioperative intravenous ketamine for acute postoperative pain in adults. Cochrane Database Syst Rev 2018;(12): CD012033.

4. Balzer N, McLeod SL, Walsh C, et al. Low-dose ketamine for acute pain control in the emergency department: a systematic review and meta-analysis. Acad Emerg Med 2021;28:444-54.

5. Loftus RW, Yeager MP, Clark JA, et al. Intraoperative ketamine reduces perioperative opiate consumption in opiate-dependent patients with chronic back pain undergoing back surgery. Anesthesiology 2010;113: 639-46. 
Intérêts concurrents : Jonathan Zipursky déclare avoir reçu des honoraires de firmes juridiques privées pour des opinions médicolégales concernant l'innocuité et l'efficacité des médicaments, indépendamment des travaux soumis. Aucun autre intérêt concurrent n'a été déclaré.

Cet article a été révisé par des pairs.

Affiliations : Département de médecine (Silverstein, Juurlink, Zipursky), Université de Toronto; Division de pharmacologie et de toxicologie clinique (Juurlink, Zipursky), Centre des sciences de la santé Sunnybrook; Institut des politiques, de la gestion et de l'évaluation de la santé (Juurlink, Zipursky), Université de Toronto; ICES Central (Juurlink), Toronto, Ont.

Propriété intellectuelle du contenu : Il s'agit d'un article en libre accès distribué conformément aux modalités de la licence Creative Commons Attribution (CC BY-NC-ND 4.0), qui permet l'utilisation, la diffusion et la reproduction dans tout médium à la condition que la publication originale soit adéquatement citée, que l'utilisation se fasse à des fins non commerciales (c.-à-d., recherche ou éducation) et qu'aucune modification ni adaptation n'y soit apportée. Voir : https://creativecommons.org/licenses/by-nc-nd/4.0/deed.fr

Correspondance : William Silverstein,

William.Silverstein@mail.utoronto.ca

Le JAMC vous invite à soumettre vos textes pour la rubrique « Cinq choses à savoir ... » en ligne à http://mc.manuscriptcentral.com/cmaj. 